\title{
Electrical Control of a Single Mn Atom in a Quantum Dot
}

\author{
Y. Léger, ${ }^{1}$ L. Besombes, ${ }^{1}{ }^{*}$ J. Fernández-Rossier, ${ }^{2}$ L. Maingault, ${ }^{1}$ and H. Mariette ${ }^{1}$ \\ ${ }^{1}$ CEA-CNRS group "Nanophysique et Semiconducteurs," Laboratoire de Spectrométrie Physique, CNRS \\ and Université Joseph Fourier-Grenoble 1, Boîte Postale 87, F-38402 St. Martin d'Hères, France \\ ${ }^{2}$ Instituto Universitario de Materiales de Alicante, Universitad de Alicante, San Vicente del Raspeig, 03690 Spain
}

(Received 14 April 2006; published 7 September 2006)

We report on the reversible electrical control of the magnetic properties of a single $\mathrm{Mn}$ atom in an individual quantum dot. Our device permits us to prepare the dot in states with three different electric charges, $0,+1 e$, and $-1 e$ which result in dramatically different spin properties, as revealed by photoluminescence. Whereas in the neutral configuration the quantum dot is paramagnetic, the electron-doped dot spin states are spin rotationally invariant and the hole-doped dot spins states are quantized along the growth direction.

DOI: 10.1103/PhysRevLett.97.107401

PACS numbers: 78.67.Hc, 75.75.+a, 78.55.Et

As the size of magneto-electronic devices scales down, it becomes increasingly important to understand the properties of a single magnetic atom in a solid state environment [1-5]. Atomic scale surface probes have been successfully used in this regard [1-3]. More recently, optical probing of both magnetic [4] and nonmagnetic [6] atoms in semiconductors has been demonstrated. Magnetically doped semiconductors have been used in the fabrication of electrically active devices that control the magnetic properties like transition temperature and coercive field [7-9]. In these devices a macroscopic number of magnetic atoms was manipulated. Here we have fabricated an electrically active device that controls the charge state of a CdTe quantum dot (QD) doped with a single Mn atom. Our single dot micro-photoluminescence measurements reveal that the magnetic anisotropy and spin configuration of the single $\mathrm{Mn}$ atom are very different depending on the charge state of the dot, which can be 0 , or $\pm 1 e$. Thereby, our device is able to tune the magnetic properties of a single $\mathrm{Mn}$ atom embedded in a QD and represents a first step in the implementation of several proposals of electrical control of the magnetism in Mn-doped quantum dots [10-13].

In this work bias controlled single-carrier charging $[14,15]$ in combination with a photodepletion mechanism [16] are used to control the charge state of individual QDs doped with a single Mn atom. The photoluminescence (PL) of neutral exciton $X[1$ electron $(e)$ and 1 hole $(h)]$, negatively charged exciton $X^{-}(2 e, 1 h)$ and positively charged exciton $X^{+}(1 e, 2 h)$ coupled with a single magnetic atom are observed in the same QD. The PL emission pattern of the charged excitons differs strongly from that of the neutral exciton. This difference reflects the fact that the Mn spin is very sensitive to the number of electrons and holes in both the excited and the ground states of the optical transitions [11,12].

Micro-spectroscopy was used to study the magnetooptical properties of self-assembled CdTe/ZnTe QDs doped with single Mn atoms. A low density of Mn atoms is introduced in the QD plane exploiting the Mn diffusion through a ZnTe spacer layer grown on a ZnMnTe barrier $[4,17]$. The low temperature $(5 \mathrm{~K}) \mathrm{PL}$ of single QDs is excited with the $514.5 \mathrm{~nm}$ line of an argon laser or a tunable dye laser and collected through aluminum shadow masks with $0.2-1.0 \mu \mathrm{m}$ apertures. The PL is then dispersed by a $2 \mathrm{~m}$ double monochromator and detected by a nitrogencooled Si charged-coupled device camera.

CdTe/ZnTe QDs are $p$-type modulation doped by the transfer of holes from the $p$-doped ZnTe substrate and from surface states that act as acceptors $[18,19]$. The occupation of the QDs by holes can be controlled by an external bias voltage $V$ on an aluminum Schottky gate with respect to a back contact on the $p$-type substrate. The bias dependent emission of a nonmagnetic QD and a Mn-doped QD is presented in Fig. 1. For increasing $V$, the surface level

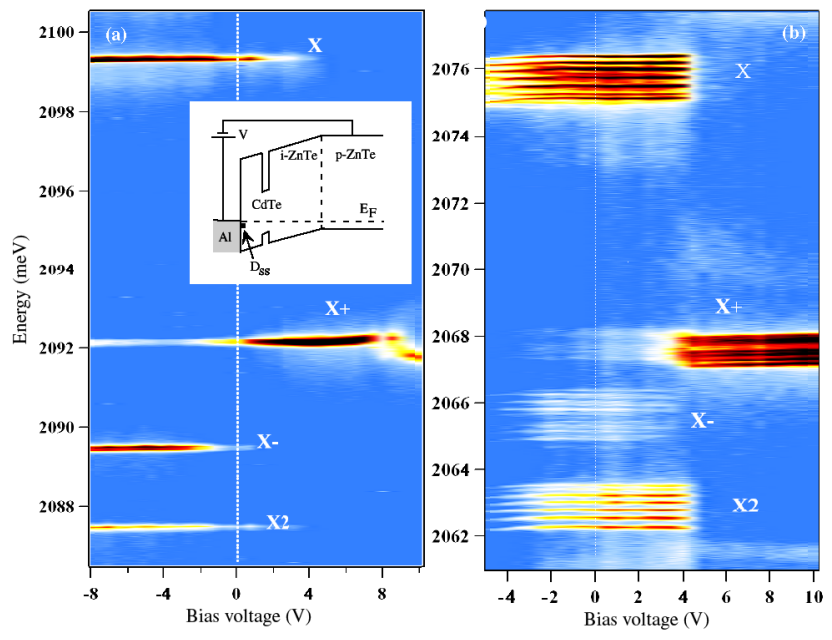

FIG. 1 (color online). Color-scale plot of the photoluminescence intensity of a nonmagnetic QD (a) and a single Mn-doped QD (b) in a Schottky structure as a function of emission energy and bias voltage. The series of emission lines can be assigned to QD $s$-shell transitions, namely, the recombination of the neutral exciton $(X)$, biexciton $\left(X^{2}\right)$, positively charged exciton $\left(X^{+}\right)$, and negatively charged exciton $\left(X^{-}\right)$. 
states are shifted below the ground hole level in the QDs which results in the single hole charging of the dots. The optically generated excitons then form charged excitons with the bias induced extra hole in the QD. At zero bias or negative bias, the Fermi level is above the ground state and the QDs are likely to be neutral. However, the separate capture of photo-created electron or holes can sometimes charge the dots so that weak contributions of $X^{+}$or $X^{-}$are observed in the zero bias spectra.

At zero bias, excess electrons can also be injected in the QD using resonant optical excitation into the QD levels. Under resonant excitation (energy below the band gap of the barriers), optical transitions from delocalized valence band states to the confined electron levels will preferentially create electrons in the QD [20]: the probability of finding an excess electron in the QD is increased. As presented in Fig. 2(a), the negatively charged exciton emission is then seen for some discrete excitation energies. After the recombination of the charged exciton $X^{-}$, a single hole is likely to be captured to neutralize the QD and create a neutral exciton. This neutralization process is responsible of the simultaneous observation of charged and neutral species under resonant excitation [Fig. 2(a)].

The charge state of the dot can also be optically tuned [Fig. 2(b)]. By combining a weak nonresonant excitation with the resonant one, a few carriers are created in the $\mathrm{ZnTe}$ barrier. They do not significantly contribute to the luminescence [lower PL spectra in Fig. 2(b)] but reduce the PL contribution of $X^{-}$in favor of the neutral species. This

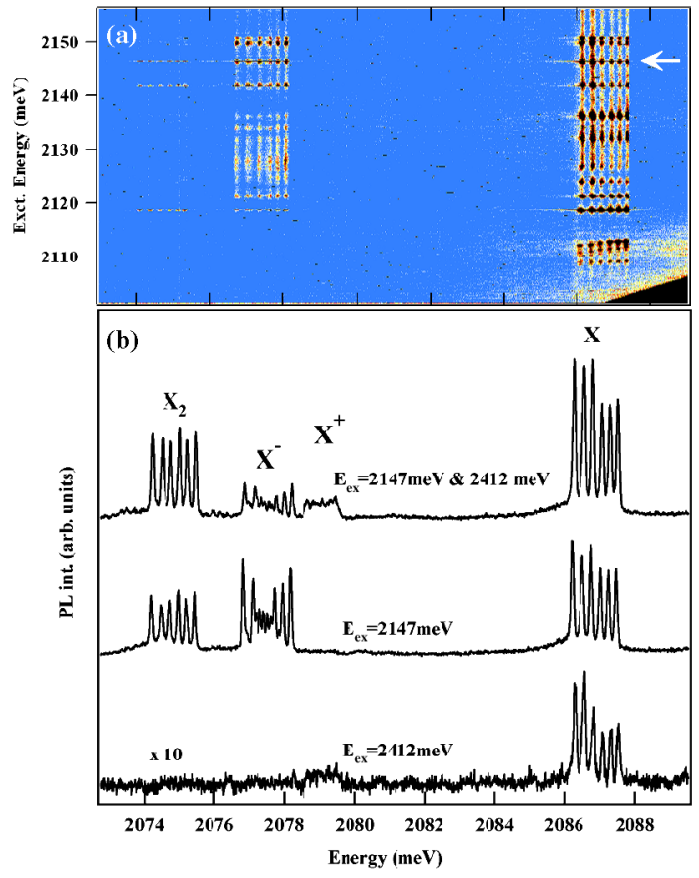

FIG. 2 (color online). (a) Multichannel photoluminescence excitation spectra of a single Mn-doped QD at zero gate voltage. (b) Detail of the PL of a single Mn-doped QD under resonant excitation $\left(E_{\mathrm{ex}}=2147 \mathrm{meV}\right)$, nonresonant excitation $\left(E_{\mathrm{ex}}=\right.$ $2412 \mathrm{meV}$ ), and both resonant and nonresonant excitation. evolution is characteristic of a photodepletion mechanism in modulation doped QDs [16]. High energy photoexcited $e-h$ pairs are dissociated in the space charge region surrounding the negatively charged QDs and neutralize the QDs.

These two charge control mechanisms (bias voltage and resonant excitation combined with photodepletion) allow us to independently probe the interaction between individual carriers (electron or hole) and an individual magnetic atom. We consider first the negatively charged exciton. Figure 3(a) presents a detail of the recombination spectrum for $X^{-}$coupled with a single $\mathrm{Mn}$ atom obtained at zero bias under resonant excitation. Eleven emission lines are clearly observed with intensity decreasing from the outer to the inner part of the emission structure.

We propose a model that accounts quantitatively for the emission spectrum shown in Fig. 3(a). The emitting state in the $X^{-}$transition has two conduction band (CB) electrons and one hole coupled to the Mn. The effect of the two spinpaired electrons on the Mn is strictly zero. Thereby, the spin structure of the $X^{-}$state is governed by the interaction of the hole with the Mn. Based upon previous work [12,2124] we propose the following Hamiltonian:

$$
\mathcal{H}_{h-\mathrm{Mn}}=I_{h}\left(S_{z} j_{z}+\frac{\epsilon}{2}\left(j_{-} S_{+}+j_{+} S_{-}\right)\right)
$$

where $\vec{S}$ is the Mn spin operator and $\vec{j}$ acts on the hole lowest energy doublet. The first term is the spin conserving or Ising exchange whereas the second is only possible if there is some heavy-light hole mixing [21,22]. From our measurements we find that $\epsilon$ is small and, in a first stage, we neglect it. Later we will show its influence. The 12 eigenstates of $\mathcal{H}_{h-\mathrm{Mn}}$ with $\epsilon=0$ are organized as six doublets [Fig. 3(b)] with well-defined $S_{z}$ and $j_{z}$ (Mn and hole spin along the $z$ axis). We label these states as $\left|S_{z}, j_{z}\right\rangle$. Recombination of one of the CB electrons with the hole of the $X^{-}$state leaves a final state with a single CB elec-

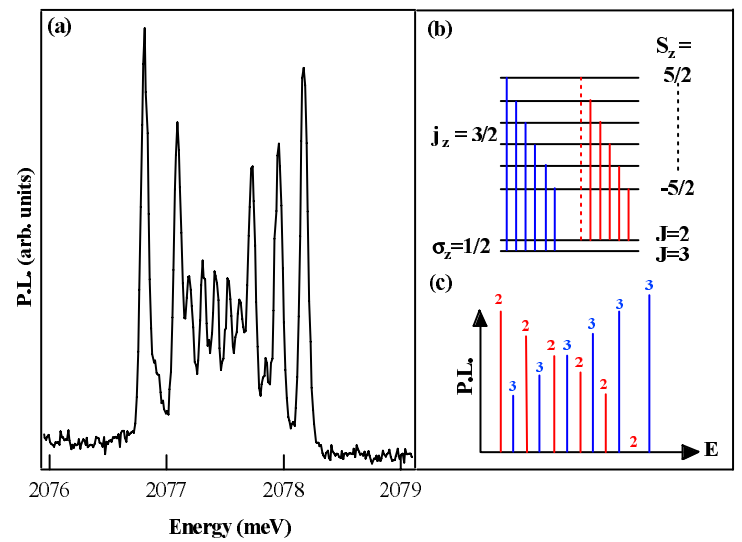

FIG. 3 (color online). (a) Detail of the unpolarized emission spectrum of a negatively charged exciton $\left(X^{-}\right)$coupled with a Mn atom. (b) Scheme of the $\sigma+$ optical transitions of $\left(X^{-}, \mathrm{Mn}\right)$ and their respective PL intensity distribution (c). 
tron coupled to the Mn. The spin Hamiltonian of this system is the ferromagnetic Heisenberg model, $\mathcal{H}_{e-\mathrm{Mn}}=$ $-I_{e} \vec{S} \cdot \vec{\sigma}_{e}$. The 12 eigenstates of the Mn-electron complex are split into a ground state septuplet (total spin $J=3$ ) and a fivefold degenerate manifold with $J=2$. We label them all as $\left|J, J_{z}\right\rangle$.

Thereby, for each of the six doublets of $X^{-}$there are two possible final states after annihilation of an $e-h$ pair, with either $J=2$ or $J=3$. From this consideration alone, we would expect 12 spectrally resolved lines. Their weight is given by both optical and spin conservation rules. Since electrons and holes reside in $s$ and $p$ bands, respectively, the $\Delta L=1$ optical selection rule is immediately satisfied. The polarization of the photon imposes an additional selection rule on $\Delta M$ which is accounted for by the spin of the electron and hole. The Mn spin is not affected by the transition.

The weight of optical transitions between the initial state $|i\rangle=(\uparrow, \downarrow)_{e} \times\left|S_{z}, j_{z}\right\rangle$ and the final state $|f\rangle=\left|J, J_{z}\right\rangle$ is proportional to $\left|\left\langle f\left|\sum_{\sigma} P\left(\sigma, j_{z}\right) c_{\sigma} d_{j_{z}}\right| i\right\rangle\right|^{2}$ where $c_{\sigma}$ annihilates a CB electron with spin $\sigma$ and $d_{j_{z}}$ annihilates a VB hole with angular momentum $j_{z}$. Here $P\left(\sigma, j_{z}\right)$ is given by the polarization selection rule.

Let us consider, for instance, $\sigma+$ recombination transitions where the $\left(\downarrow_{e}, \Uparrow_{h}\right) e-h$ pair is annihilated. Each of the six doublets, characterized by their Mn spin projection $S_{z}$, can be an initial state. After the electron-hole annihilation, the resulting state is $\left|S_{z}, \uparrow_{e}\right\rangle$ which, in general, is not an eigenstate of $\mathcal{H}_{e, \mathrm{Mn}}$. The intensity of the optical transition to a given final state $\left|J, J_{z}\right\rangle$ is proportional to the overlap $\left\langle J, J_{z} \mid S_{z}, \uparrow_{e}\right\rangle$, which is nothing but a ClebschGordan coefficient. The highest energy transition, with $\sigma+$ polarization, would correspond to the initial state $(\uparrow, \downarrow)_{e} \times\left|+5 / 2, \Uparrow_{h}\right\rangle$ and a low energy final state $\left|J=3, J_{z}\right\rangle$. After the photon emission the state of the system is $\mid S_{z}=$ $\left.+5 / 2, \uparrow_{e}\right\rangle$ which is identical to $\left|J=3, J_{Z}=+3\right\rangle$ and thereby gives the highest optical weight [Fig. 3(b)]. In contrast, emission from that initial state to $\left|J=2, J_{z}\right\rangle$ is forbidden. The other five doublets have optical weights lying between $1 / 6$ and $5 / 6$ with both $\left|J=2, J_{z}\right\rangle$ and $\left|J=3, J_{z}\right\rangle$ final states. Thereby, the number of spectrally resolved lines in this model is 11 .

The relative weight of the emission lines is accounted for by the model. According to the final state, the transitions belong to either the $J=2$ or the $J=3$ series. As the initial $S_{z}$ decreases, the overlap of $\left|\uparrow_{e} S_{z}\right\rangle$ to the $J=3(J=2)$ states decreases (increases). As presented in Fig. 3(c), the PL of $X^{-}$can be seen as a superposition of two substructures: six lines with intensities increasing with their energy position (transitions to $J=3$ states) and five lines with intensities decreasing with increasing their energy position (transitions to $J=2$ states).

Reversing the role of the initial and final states, and neglecting the small coupling of two holes to the Mn spin [25], this model should account for the emission from $X^{+}$states. Actually, different energy splittings are observed for the different excitonic species in the same QD, namely $\Delta E_{X}=1.23 \mathrm{meV}, \Delta E_{X_{-}}=1.36 \mathrm{meV}$ and $\Delta E_{X+}=0.95 \mathrm{meV}$. The energy splitting is mostly due to the Mn-hole exchange coupling, which in turn is inversely proportional to the volume of the hole wave function. The difference between $\Delta E_{X+}$ and $\Delta E_{X-}$ indicates that a significant fraction of the confinement of the hole comes from the Coulomb attraction of the 2 electrons in the initial state of the $X^{-}$emission. In contrast, in the final state of the $X^{+}$ emission there is no electron to attract the hole, resulting in a spread of the hole wave function and a smaller exchange energy. This difference appears directly in the emission structure shown in Fig. 2(b), where the peak structure of $X^{+}$is not well resolved.

In Fig. 4(a) and 4(b) we show the intensity of both $X^{+}$ and $X^{-}$emission as a function of the direction of a linear analyzer. It is apparent that the central lines are linearly polarized. This polarization can only be understood if we allow for some spin-flip interaction between the Mn and

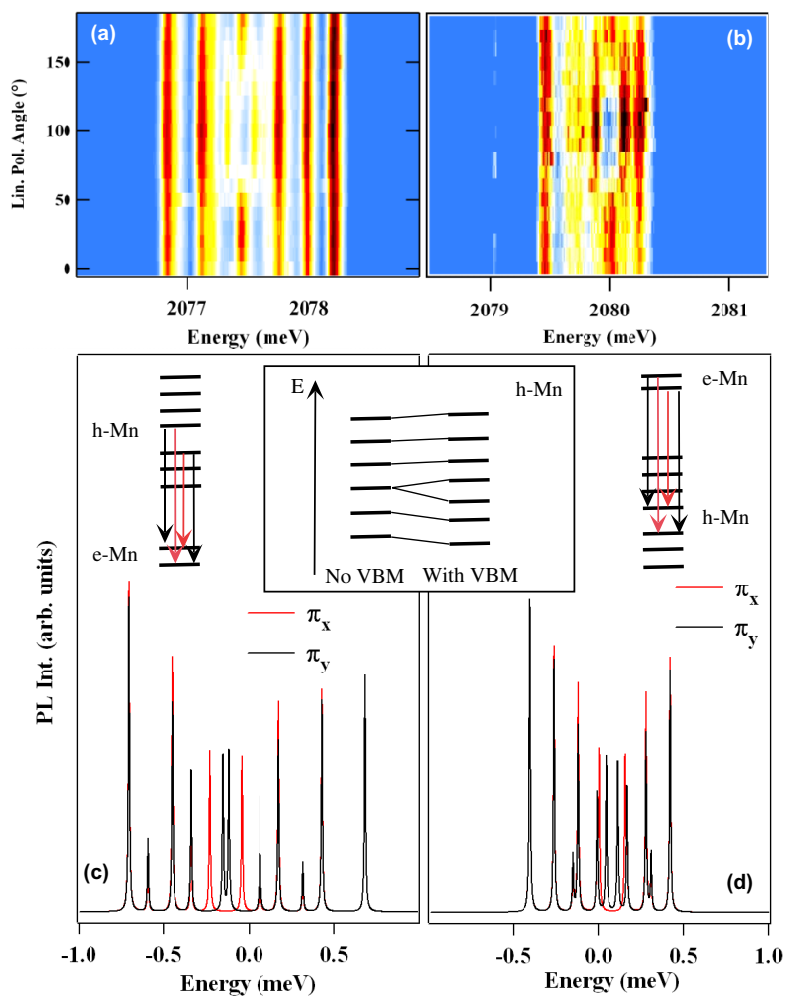

FIG. 4 (color online). Color-scale plot of the dependence of the PL of $\left(X^{-}, \mathrm{Mn}\right)(\mathrm{a})$ and $\left(X^{+}, \mathrm{Mn}\right)(\mathrm{b})$ on the direction of a linear analyzer, in the same QD. Three lines in the center of the structure are linearly polarized. (c) and (d) calculated linearly polarized PL spectra of $\left(X^{-}, \mathrm{Mn}\right)$ and $\left(X^{+}, \mathrm{Mn}\right)$ with exchange integrals $I_{e}$ and $I_{h}$ chosen to reproduce the overall splitting for $X^{+}$and $X^{-}$presented in (a) and (b). Transitions are arbitrarily broadened by $10 \mu \mathrm{eV}$. The schemes in (c) and (d) show energy levels involved in $\left(X^{-}, \mathrm{Mn}\right)$ and $\left(X^{+}, \mathrm{Mn}\right)$ recombination with valence band mixing. The central inset presents the energy level scheme of the $h$-Mn system without and with valence band mixing (VBM). 
the hole [second term in Eq. (1)]. Provided that $\epsilon \ll 1$, the effect of this interaction is small both on the wave function and on the degeneracy of all the doublets except the third, which is split, as illustrated in the inset of Fig. 4. The split states are the bonding and antibonding combinations of $\left|S_{z}=-1 / 2, \Uparrow_{h}\right\rangle$ and $\left|S_{z}=+1 / 2, \Downarrow_{h}\right\rangle$. These states are coupled, via linearly polarized photons, to the $|2,0\rangle$ and $|3,0\rangle e-\mathrm{Mn}$ complex, and four linearly polarized lines should be observed on the emission spectra as shown in the insets of Fig. 4(c) and 4(d). Polarization directions are controlled by the distribution of strains through the BirPikus Hamiltonian [22,26].

Although small, the spin-flip interaction has an important consequence: the structure of the positively and negatively charged exciton recombination are no longer symmetric. In the case of $X^{-}$(left panels of Fig. 4) the $h$-Mn structure occurs in the initial state, whereas in the case of $X^{+}$(right panels of Fig. 4) it matters in the final state. The linearly polarized transitions appear on the (comparatively) low energy side of the structure for $\left(X^{-}\right.$, $\mathrm{Mn})$ and on the (comparatively) high energy side for $\left(X^{+}, \mathrm{Mn}\right)$. This characteristic structure gives an additional way of identifying the different charged species. Yet another interesting feature of these linearly polarized transitions: both the initial and final states in the four lines are entangled states of the form $\left|S_{z}=+1 / 2\right\rangle \times$ $|\downarrow\rangle_{f} \pm\left|S_{z}=-1 / 2\right\rangle \times|\uparrow\rangle_{f}$ where $f$ stands for either electron or hole.

We have obtained numerical values of $I_{h}, I_{e}$, and $\epsilon$ for the charged excitons comparing the transition probabilities calculated with our model (1) [Fig. 4(c) and 4(d)] to the experimental data. The electron-Mn exchange integral $I_{e}$ is deduced from magneto-optics measurements on the neutral exciton [4]. $I_{h}$, the hole-Mn exchange integral, is then chosen to reproduce the overall splitting of the two charged species emission structure. There is a good agreement between the experimental data and our model with $I_{e}=$ $40 \mu \mathrm{eV}, \quad I_{h}(X)=150 \mu \mathrm{eV}, \quad I_{h}\left(X^{+}\right)=95 \mu \mathrm{eV}, \quad$ and $I_{h}\left(X^{-}\right)=170 \mu \mathrm{eV}$. The different values of $I_{h}$ directly reflect the expected variation of the confinement of the hole. The main characteristics of the emission spectra are well reproduced, namely, the number of emission lines, their intensity distribution, and the linear polarization structure, with a slight valence band mixing coefficient $\epsilon=0.05$. This small value of the valence band mixing coefficient shows that hole-Mn exchange interaction remains highly anisotropic.

In summary, we have fabricated a device with electrically tunable magnetic properties, in analogy with two dimensional dilute magnetic semiconductor based electri- cally active heterostructures [7-9], but scaling the number of controlled magnetic atoms down to one and the size of the active region down to a few nanometers. Our micro-PL experiments permit us to identify three magnetically different ground states corresponding to three charge states $( \pm 1 e$ and 0$)$ and to measure the exchange interaction of both a single electron and a single hole with a single magnetic atom. The final state of $\left(X^{+}, \mathrm{Mn}\right)$ transition $(1 h, 1 \mathrm{Mn})$ is nondegenerate in the absence of external magnetic field. This splitting of the different spin configurations should efficiently increase the spin relaxation time of both $\mathrm{Mn}$ and holes. This property could be exploited to store digital information on a single atom.

This work is supported by ANR-France Contract MOMES. J.F.-R. acknowledges funding from MECSpain (Grants No. FIS200402356 and Ramon y Cajal) and from Generalitat Valenciana (No. GV05-152).

*Electronic address: lbesombes@ @pectro.ujf-grenoble.fr

[1] H. C. Manoharan et al., Nature (London) 403, 512 (2000).

[2] P. Gambardella et al., Science 300, 1130 (2003).

[3] A. M. Yakunin et al., Phys. Rev. Lett. 92, 216806 (2004).

[4] L. Besombes et al., Phys. Rev. Lett. 93, 207403 (2004).

[5] A. J. Heinrich et al., Science 306, 466 (2004).

[6] F. Jelezko et al., Phys. Rev. Lett. 92, 076401 (2004).

[7] H. Ohno et al., Nature (London) 408, 944 (2000).

[8] H. Boukari et al., Phys. Rev. Lett. 88, 207204 (2002).

[9] D. Chiba et al., Science 301, 943 (2003).

[10] Al. L. Efros et al., Phys. Rev. Lett. 87, 206601 (2001).

[11] J. Fernández-Rossier and L. Brey, Phys. Rev. Lett. 93, 117201 (2004).

[12] A. O. Govorov and A. V. Kalameitsev, Phys. Rev. B 71, 035338 (2005); A. O. Govorov, ibid. 72, 075359 (2005).

[13] J.I. Climente et al., Phys. Rev. B 71, 125321 (2005); F. Qu and P. Hawrylak, Phys. Rev. Lett. 95, 217206 (2005);

F. Qu and P. Hawrylak, ibid. 96, 157201 (2006).

[14] R. J. Warburton et al., Nature (London) 405, 926 (2000).

[15] J. Seufert et al., Appl. Phys. Lett. 82, 3946 (2003).

[16] A. Hartmann et al., Phys. Rev. Lett. 84, 5648 (2000).

[17] F. Tinjod et al., Appl. Phys. Lett. 82, 4340 (2003).

[18] W. Maslana et al., Appl. Phys. Lett. 82, 1875 (2003).

[19] S. Bhunia et al., J. Appl. Phys. 87, 2931 (2000).

[20] A. Vasanelli et al., Phys. Rev. Lett. 89, 216804 (2002).

[21] J. Fernández-Rossier, Phys. Rev. B 73, 045301 (2006).

[22] A. V. Koudinov et al., Phys. Rev. B 70, 241305 (2004).

[23] F. V. Kyrychenko et al., Phys. Rev. B 70, 205317 (2004).

[24] Y. Léger et al., Phys. Rev. B 72, 241309 (2005).

[25] L. Besombes et al., Phys. Rev. B 71, 161307 (2005).

[26] M. Tadic et al., Phys. Rev. B 65, 165333 (2002) 